Revue d'histoire de l'Amérique française

REVUE D.HISTOIRE DE L'AMÉRIQUE FRANÇAISE

\title{
Un village d'Abénakis sur la rivière Missisquoi
}

\section{Thomas Charland}

Volume 15, numéro 3, décembre 1961

URI : https://id.erudit.org/iderudit/302132ar

DOI : https://doi.org/10.7202/302132ar

Aller au sommaire du numéro

Éditeur(s)

Institut d'histoire de l'Amérique française

ISSN

0035-2357 (imprimé)

1492-1383 (numérique)

Découvrir la revue

Citer cet article

Charland, T. (1961). Un village d'Abénakis sur la rivière Missisquoi. Revue

d'histoire de l'Amérique française, 15(3), 319-332.

https://doi.org/10.7202/302132ar d'utilisation que vous pouvez consulter en ligne.

https://apropos.erudit.org/fr/usagers/politique-dutilisation/ 


\section{UN VILLAGE D'ABÉNAKIS SUR LA RIVIËRE MISSISQUOI *}

Après la paix d'Utrecht (1713), les Abénakis domiciliés à Saint-François et à Bécancour furent tentés de retourner dans leur pays d'origine, le long des rivières Kennebec et Pentagoët (ou Penobscot). Ils venaient de perdre un de leurs principaux moyens de subsistance, la guerre, et par ailleurs, ils étaient invités par leurs frères vivant du côté de l'Acadie à prendre part au commerce plus lucratif que ceux-ci pratiquaient avec leurs voisins de la Nouvelle-Angleterre. La tentation dura une bonne quinzaine d'années. Conformément aux directives reçues de la Cour de Versailles, le gouverneur et l'intendant de la Nouvelle-France firent tout pour les retenir, allant même jusqu'à menacer ceux de Narantsouak de les priver d'un missionnaire.

Par contre, ces mêmes autorités les laissèrent aller s'établir, vers 1732 , à quelques milles de l'entrée de la rivière Missisquoi, près de l'endroit où s'élève aujourd'hui la ville de Swanton, dans l'Etat du Vermont. Ils s'y construisirent un village, qu'ils entourèrent d'une palissade. Un moment, on songea à fonder une mission spéciale pour eux. Le gouverneur et l'intendant firent bâtir une résidence pour le missionnaire qui les visitait. Ils auraient voulu le gratifier aussi d'une chapelle; mais diverses circonstances firent ajourner l'exécution de ce projet. ${ }^{1}$

Ce sont sans doute les besoins de la chasse qui avaient incité les Abénakis à cet éloignement, mais c'est aussi, on peut bien

\footnotetext{
* Ces pages sont extraites d'un ouvrage en préparation sur les Abénakis de Saint-François.

1 En 1909, à l'occasion des fêtes commémoratives du troisième centenaire de la découverte du lac Champlain, on a érigé, à Swanton, un monument sur lequel se lit l'inscription suivante: «Near this spot stood the first church erected in Vermont about 1700 by the Jesuit Fathers to the glory of God Almighty for the Mission of St Francis. 》 Je ne connais aucun document autorisant pareille affirmation. Je trouve même invraisemblable qu'on aît permis aux Abénakis de s'établir sur la rivière Missisquoi à cette époque, puisque, en 1704, le gouverneur Vaudreuil avait renoncé à son projet de les établir aux environs de Chambly, et cela pour ne pas porter ombrage aux Iroquois.
} 
le penser, le désir de se rapprocher des comptoirs anglais pour y porter leurs pelleteries, et même celles des Français. Ils chassaient déjà dans cette région, de temps immémorial. ${ }^{2}$ Et comme ce territoire était fréquenté aussi par les Iroquois, il avait dû se produire de sanglantes rencontres entre ces deux nations ennemies. A ce propos, l'abbé J.-A. Maurault raconte l'événement suivant:

Dans le cours de l'été 1695 , un parti d'Iroquois se réfugia un jour sur une petite île du lac Champlain, pour y passer la nuit. Cinq ou six Abénakis, occupés à faire la chasse près de ce lac, les ayant apercus, résolurent aussitôt de les attaquer. Mais il fallait auparavant prendre quelque connaissance du lieu où étaient ces ennemis. Ils envoyèrent donc l'un d'eux dans ce but. L'Abénakis se rendit à l'île, à la nage; puis, se glissant le plus légèrement possible, à travers les herbes et les broussailles, il alla se cacher à quelques pas du campement ennemi, de manière à voir ce qui s'y passait et à entendre ce qui s'y disait. Trente Iroquois étaient assis autour d'un feu et formaient des projets de vengeance contre les Abénakis, tandis qu'une énorme tête de bœuf, qui devait être le mets de leur repas, cuisait suspendue au-dessus du feu. Après le repas, l'un

2 «e the Misiskoui Indians of the St Francis or Abenaki Tribe have inhabited that part of Lake Champlain known by the Name of Misiskoui Time unknown to any of us here present. "Speech of the Misiskoui Indians to the Governor of Quebec in the North End of Lake Champlain, $8^{\text {th }}$ September 1766. $\gg$ Archives canadiennes, série Q, 3: 328. Texte publié par George H. Montgomery, Missisquoi Bay (Granby, 1950), 14, et par John C. Huden, «Indian Troubles in early Vermont», Vermont History, 26 (1958) : 38-39. Deux autres documents conservés aux Archives canadiennes, Indian Records, Series II, 9: 84-87 et 14:111, précisent que la rencontre des Abénakis avec le Gouverneur eut lieu à l'Isle Lamotte. «Plusieurs sauvages Abénakis qui étaient en chasse autour du lac Champlain tardaient à venir. 》 Vaudreuil au ministre, 27 avril 1709, Rapport de l'Archiviste de la Province de Québec (1942-1943), 399. — «A Misiskoui et tout le lac Champlain où les Abénakis et les Iroquois vont en chasse. \ Le Chevalier Raymond, «Mémoire sur les postes du Canada 》 (1754), RAPQ (1927-1928), 343. - Le nom même de Missisquoi serait d'origine abénakise et viendrait de Massipskoik, qui signifie où il y a de la pierre à fusil. Joseph Laurent, New familiar Abenakis and English Dialogues (Québec, 1884), 216; John C. Huden, Indian Place Names in Vermont (1957), 21. - Les Français prononcèrent d'abord Missiskoui et Michiscoui. D. Girouard, «L'Etymologie du mot Missisquoi 》, Bulletin des recherches historiques, 11 (1905) : 270-277, et « Origine du mot Missisquoi », ibid., 12 (1906) : 33-37. 
d'eux amassa les os et les jeta loin derrière lui, en s'écriant: "Puissions-nous dévorer tous ces chiens d'Abénakis, comme cette tête, et les traiter ainsi !» Bientôt, ils se couchèrent autour du feu et ne tardèrent pas à s'endormir profondément. L'Abénakis se glissa alors jusqu'aux canots des ennemis, y pratiqua, avec son couteau, de larges ouvertures, puis retourna vers ses frères. Les Abénakis se rendirent aussitôt dans l'île, profitant du sommeil de leurs ennemis, se jetèrent sur eux la hache à la main, en poussant d'horribles cris. Ils en tuèrent plusieurs sur le champ. Les autres, saisis d'une terreur panique, et se croyant attaqués par un grand nombre d'Abénakis, se précipitèrent dans leurs canots pour s'enfuir, et se noyèrent. Les Abénakis nommèrent cette île "Otepsek 》, l'île-à-la-tête, à cause de la tête de bœuf. ${ }^{3}$

La construction, en 1731, du fort Saint-Frédéric, à la Pointeà-la-Chevelure (Crown Point pour les Anglais), au sud du lac Champlain, fut suivie, à partir de 1733, d'une série de concessions de seigneuries autour de la baie Missisquoi, région jusque-là inhabitée à cause du voisinage des Anglais et des Iroquois. L'octroi de ces seigneuries avait pour but de sortir le fort de son isolement. Ce but ne fut pas atteint. Les concessionnaires étaient pour la plupart des fonctionnaires gouvernementaux ou des officiers militaires en service du commencement à la fin de l'année, qui d'ailleurs préféraient l'exploitation des forêts à la colonisation des terres. ${ }^{4}$ Leurs seigneuries, n'ayant pas été mises en valeur, furent réunies au domaine du Roi. C'est ce qui arriva notamment pour les seigneuries qui avaient été concédées au capitaine Paul-Louis Dazemard de Lusignan (6 avril 1733)

3 Histoire des Abénakis (Sorel, 1866), 230-231. Voir aussi HenryLorne Masta, Abenaki Indian Legends, Grammar and Place Names (Victoriaville, 1932), 33-34. - Cette île est située vis-à-vis l'embouchure de la rivière Lacolle et porte aujourd'hui le nom de $A s h$ Island. Les Canadiens l'appelaient l'Ile-aux-Têtes. Dans ses Voyages et mémoires sur le Canada (Québec, 1889), 63, Franquet donne une explication un peu différente de cette appellation: "On nomme telles ces isles (grande isle aux Têtes et petite isle aux Têtes) à cause d'un massacre de 14 Agniers par des Francais qui exposèrent leurs têtes au bout de piques. »

4 Pierre-Georges Roy, Hommes et choses du Fort Saint-Frédéric (Montréal, 1946), 314. 
et à Philippe-René LeGardeur de Beauvais fils (20 juillet 1734). Le territoire de ces deux seigneuries fut concédé de nouveau, le 23 septembre 1748, sous le nom de seigneurie de Saint-Armand, à Nicolas-René Levasseur, constructeur des vaisseaux du Roi à Québec.

Dans l'acte de concession de la seigneurie de Beauvais, dont faisait partie la presqu'île où débouche la rivière Missisquoi, il n'était pas question des Abénakis. Il n'en fut pas davantage dans celui de la seigneurie de Saint-Armand; mais il semble que, cette fois, on aît tenu compte de l'occupation, sinon de la possession de leur territoire par les Abénakis. Cette seigneurie, en effet, avait un front de six lieues, trois de chaque côté de la rivière Missisquoi, à prendre, non de l'embouchure de cette rivière, mais « à huit arpents au-dessous de la première chute qui se trouve à trois lieues dans la profondeur de la dite rivière en remontant ${ }^{5}, 5$ soit en arrière du territoire réservé aux Abénakis.

Cette région était riche en bois propres à la construction des navires, au rapport de Levasseur, qui l'avait visitée en 1744 et en $1745 .^{6}$ L'intendant Hocquart avait alors écrit au ministre: « J'avais projeté au commencement de l'hiver de faire faire un moulin à scie dans le fond de la rivière de Michiscouy, il s'y trouve une chute fort propre pour un pareil établissement et dans le voisinage une belle et grande pinière de pins blancs d'une qualité fort supérieure aux arbres de cette espèce répandus dans le reste du pays. J'en ai remis l'exécution à un temps plus favorable; cet établissement servirait encore à exploiter à moindres frais des bordages de chêne que l'on pourrait tirer du même endroit pour le service de la marine. $~^{7}$

C'était alors la guerre de la Succession d'Autriche. Celle-ci terminée, ce fut Levasseur qui exécuta le projet d'Hocquart. Une fois en possession de sa seigneurie, il bâtit, à ses frais, un

5 P.-G. Roy, Inventaire des concessions en fief et seigneurie ... (6 vol., Beauceville, 1927-1929), 5: 62 .

6 Levasseur à Hocquart, 6 mars 1744, et « Journal d'un voyage dans le lac Champlain pour $\mathrm{y}$ faire couper des mâts de vaisseaux $\gg, 22$ février $1745, \mathrm{AC}, \mathrm{C} 11 \mathrm{~A}, 81$ et 83.

7 Hocquart au ministre, 16 octobre 1745, AC, C11A, 83. 
moulin sur la rivière Missisquoi « pour fabriquer des planches $\gg .8$ S'il faut en croire les Abénakis, le gouverneur et l'intendant se seraient rendus sur les lieux pour choisir le site du moulin, auraient réservé une lieue carrée pour sa construction et pour la coupe du bois, et auraient obtenu leur consentement à la condition qu'on leur donne les planches dont ils pourraient avoir besoin. ${ }^{9}$

Les Abénakis s'étaient déjà installés sur la rivière Missisquoi, dès avant 1733. Dans son volume sur les Hommes et choses du Fort Saint-Frédéric, Pierre-Georges Roy donne la liste des baptêmes, mariages et sépultures faits par les aumôniers du fort. ${ }^{10}$ J'y ai relevé 60 baptêmes et 20 sépultures d'Abénakis, entre 1733 et 1758. Les aumôniers mentionnent leur lieu d'origine: SaintFrançois et Bécancour. Ce qui, évidemment, ne veut pas dire que les Abénakis partaient de Saint-François et de Bécancour, où ils avaient leurs missionnaires, pour aller faire baptiser et inhumer au Fort Saint-Frédéric, à quelque deux cents milles de chez eux; mais que les Abénakis de Saint-François et de Bécancour établis sur la rivière Missisquoi allaient, pour les besoins religieux, au Fort Saint-Frédéric, le poste le plus facile d'accès pour eux, sinon le plus rapproché. On y entretenait un interprète de la langue abénakise, aux appointements de 300 francs. ${ }^{11}$

Je m'étonne que P.-G. Roy, qui a bourré son volume de tous les renseignements qu'il a pu recueillir sur les hommes et les

8 Mémoire de Levasseur au ministre, 25 février 1754, AC, C11A, 99. Dans ce mémoire, Levasseur demandait que, en considération des dépenses qu'il avait faites pour la construction de son moulin, on lui accordât la préférence pour la fourniture des planches aux chantiers du Roi à Québec. Le ministre écrivit à l'intendant Bigot, le 14 juin 1754, qu'on pouvait prendre ces planches, si elles étaient aussi bonnes que les autres et pourvu que le Roi ne fût pas lésé par rapport aux prix. AC, B 99. - Joseph-Noël Fauteux, Essai sur l'industrie au Canada sous le régime français (Québec, 1927), 274.

9 «Speech of the Misiskoui Indians...» Ce discours manque d'exactitude: il donne comme gouverneur d'alors (1748) Vaudreuil, au lieu de La Galissonière !

10 D'après une copie des registres du fort qui se trouve aux Archives canadiennes à Ottawa, Série M, 200, et qui a été faite sur l'original par N.-E. Dionne. E.-Z. Massicotte, « Les Actes de mariage du Fort SaintFrédéric $\gg, B R H, 28(1921): 261$.

11 Franquet, Voyages et mémoires sur le Canada, 75. 
choses du Fort Saint-Frédéric, ne se soit pas demandé comment il se fait que des Abénakis se soient adressés si souvent aux aumôniers du fort, et qu'il n'ait pas signalé leur établissement sur la rivière Missisquoi. Il faut dire que les documents concernant cet établissement sont plutôt rares. Il en est un, pourtant, que Roy lui-même avait déjà publié dans son Inventaire des Concessions en fief et seigneurie $(5: 252)$ et qui nous apprend qu'en 1738 il y avait déjà plus de vingt cabanes d'Abénakis à Missisquoi. Ce document est une requête adressée au ministre, le comte de Maurepas, en octobre 1738, par Jean-Baptiste JutrasDesrosiers, pour se plaindre d'un empiétement des Abénakis de Saint-François sur son fief de Lussaudière. Sous prétexte que ses Abénakis viendraient à manquer de bois de chauffage ${ }^{12}$ si Jutras faisait de nouveaux établissements sur la partie arrière de son fief, le Père Joseph Aubery, s.j., avait obtenu du gouverneur des Trois-Rivières, Pierre de Rigaud de Vaudreuil, une défense à Jutras de mettre à exécution son projet de concession jusqu'à ce que le gouverneur général en eût décidé. Le Père Aubery était sans doute au courant des intentions des autorités sur cette partie du fief de Lussaudière. On lit dans le « Mémoire $\mathrm{du} \mathrm{S}^{\mathrm{r}}$ de Catalogne sur les seigneuries » (1715) : « La seigneurie de Luçaudière appartient au sieur Raimbeau, procureur du Roi à Montréal, il y a peu d'habitants par rapport aux terres basses, et la plupart des meilleures sont destinées pour l'établissement des Abénakis. Il y a toute sorte de bois, bonne chasse et pêche. ${ }^{13}$

A la suite de cette défense, les Abénakis s'étaient pour ainsi dire emparés de plus de cinquante arpents de front sur une lieue de profondeur dans cette partie du fief de Jutras, la meilleure puisque le reste ne pouvait être ensemencé que très tard, étant

\footnotetext{
12 Ils n'en manquaient pas vers 1736 , si on en juge par ce passage d'un document du 15 octobre de cette année-là: "Les Sauvages Abénakis de Saint-François qui sont établis, il $\mathrm{y}$ a plus longtemps que les Iroquois du Sault Saint-Louis, et qui sont plus nombreux, ne manquent ni de terres, $n i$ de bois, quoique la seigneurie de Saint-François n'ait que deux lieues de front sur deux lieues de profondeur, et sur laquelle il $\mathrm{y}$ a plusieurs habitants qui ont des concessions.» \& Réponse aux objections que les Révérends Pères Jésuites font naître pour s'opposer à la concession accordée au Sr de Boisclerc, derrière le Sault Saint-Louis 》, AC, C11A, 66; P.-G. Roy, Inventaire des concessions en fief et seigneurie, $5: 294$.
} 13 AC, F3. 
noyé presque entièrement à chaque printemps. Le gouverneur Beauharnois jugea à propos de ne pas se prononcer sur le litige et conseilla à Jutras de s'adresser à Versailles, au ministre de la marine, de qui relevaient les affaires du Canada. Dans sa requête à ce dernier, Jutras demandait d'être maintenu dans la propriété et la jouissance de la totalité de son fief, ou, s'il en était autrement ordonné, de faire limiter à une demi-lieue de profondeur le terrain qui serait laissé aux Abénakis, et, dans ce cas, de lui accorder un dédommagement proportionné à la perte qu'il encourrait, et enfin d'ordonner que lorsque les Abénakis abandonneraient ce terrain, celui-ci retournerait à son premier propriétaire, « et non aux bons Pères Jésuites ».

Jutras avait cru plaider habilement sa cause en disant que, sans l'obstination du Père Aubery à vouloir les retenir à SaintFrançois, tous les autres Abénakis de ce village se seraient transportés à la baie Missisquoi, ce qui aurait été d'un grand avantage pour établir les terres du lac Champlain et pour secourir au besoin le Fort Saint-Frédéric. Cette affirmation piqua la curiosité du ministre. Il s'enquit auprès de Beauharnois et Hocquart, ${ }^{14}$ et il apprit d'eux que les dires de Jutras n'étaient fondés que sur les discours de quelques Abénakis, discours qui n'avaient pas tiré à conséquence..$^{15}$

Le ministre fit savoir à Jutras que la volonté du Roi était de ne pas priver les Abénakis de Saint-François de prendre du bois sur une partie du fief de Lussaudière, et de ne pas dédommager le propriétaire de ce fief. Si Jutras persistait dans sa réclamation, des mesures seraient prises pour faire réunir au domaine du Roi la partie en question, puisqu'il n'avait pas rempli les conditions d'établissement attachées à sa concession. ${ }^{16}$

A Versailles, on n'était pas d'humeur à apprécier les avantages que Jutras avait fait valoir d'un éventuel exode des Abénakis

14 Le président du Conseil de Marine à Beauharnois et Hocquart, 21 avril $1739, \mathrm{AC}, \mathrm{B} 68$.

15 Beauharnois et Hocquart au ministre, 30 octobre 1739, AC, C11A, 71.

16 Le président du Conseil de Marine à Beauharnois et Hocquart, 16 avril 1741, AC, B 72; Beauharnois et Hocquart au ministre, 26 octobre 1741, AC, C11A, 75. P.-G. Roy, Inventaire des concessions en fief et seigneurie, $5: 253$. 
vers Missisquoi. On travaillait alors à mettre fin au commerce de contrebande qui se pratiquait entre le Canada et les colonies anglaises, surtout par la voie du lac Champlain. Des Français allaient porter leurs castors aux Anglais qui les leur payaient au moins un tiers plus cher que la Compagnie des Indes, et ils revenaient avec des marchandises anglaises de fabrication supérieure ou de prix moins élevé que celles du Canada: pièces de drap, d'indienne, de mousseline, d'écarlatine, chaudières, bassins et tasses d'étain, cuillers, paires de cardes à laine, fil à rets, etc. ${ }^{17}$

Ce commerce avait été presque complètement arrêté au moment de la construction du Fort Saint-Frédéric; mais il avait repris de plus belle par la suite. ${ }^{18}$ Les fraudeurs, ne pouvant plus aller eux-mêmes à Orange (Albany), avaient pris l'habitude d'y envoyer les Sauvages domiciliés, à qui on avait toujours laissé toute liberté. Le gouverneur et l'intendant se contentèrent tout d'abord de faire expliquer aux Sauvages, par leurs missionnaires, combien leur conduite était préjudiciable aux intérêts de la colonie, et de les menacer de la privation des bienfaits du Roi s'ils continuaient à enfreindre ses ordres. Les Abénakis de Saint-François leur firent répondre qu'ils avaient bien porté aux Anglais quelques castors français, mais pour avoir quelques marchandises de peu de conséquence; qu'ils avaient vu bien des Sauvages porter quantité de paquets, mais qu'eux-mêmes n'étaient pas du nombre. ${ }^{19}$ La politique de persuasion ne donna aucun résultat. Alors le gouverneur et l'intendant adoptèrent un système de contrôle. Ils proposèrent de placer un commandant au village des Abénakis et de rétablir une garnison au Sault SaintLouis. Ils durent toutefois renoncer à cette mesure, pour ne pas susciter de sentiments de défiance chez ces Sauvages. ${ }^{20}$ Instruction fut donnée au commandant du Fort Saint-Frédéric

17 Ce sont les articles qui figurent dans les saisies mentionnées par P.-G. Roy, Inventaire des ordonnances des intendants de la Nouvelle-France (4 vol., Beauceville, 1919), 2 : passim.

18 Le gouverneur et l'intendant attribuèrent cette reprise en partie à la mauvaise qualité des écarlatines françaises. Les Sauvages préféraient d'ailleurs les écarlatines bleues.

19 Beauharnois et Hocquart au ministre, 8 novembre 1737, AC, C11A, 67.

20 Le président du Conseil de Marine à Beauharnois et Hocquart, 21 avril $1739, \mathrm{AC}, \mathrm{B} 68$. 
d'empêcher les Sauvages allant à Orange de passer plus de pelleteries que n'en produisait leur propre chasse. On en vint même à exiger d'eux un certificat de leurs missionnaires attestant que ces pelleteries leur appartenaient. ${ }^{21}$

Même contrôlé, le commerce pouvait favoriser le dessein des Anglais de gagner l'amitié des Abénakis, ou du moins de s'assurer leur neutralité en cas de guerre. Pour empêcher ce résultat, et puisque les Abénakis étaient plus attachés à leur foi catholique qu'aux intérêts des Français, on eut recours à l'influence de la religion. En 1743, on décida de fonder une mission abénakise à Missisquoi. Elle contribuerait en même temps, pensait-on, à l'établissement de la région en fournissant les secours spirituels aux nouveaux colons. On espérait aussi y attirer une partie des Loups des environs d'Orange, qui avaient paru vouloir s'y retirer. Un Jésuite, le Père Étienne Lauverjat, ancien missionnaire de Saint-François et de Panaouamské, fut assigné à la nouvelle mission. On comptait sur lui pour détacher les Abénakis des Anglais et pour informer les autorités de leur conduite. ${ }^{22}$

Beauharnois et Hocquart lui firent construire une maison de pièces sur pièces et lui achetèrent des meubles et des ustensiles, le tout au coût de 1500 livres. ${ }^{23}$ Mais, quand il insista pour qu'on lui bâtît aussi une chapelle, il se heurta à la consigne si souvent répétée alors par le Roi aux autorités de la colonie, de pratiquer la plus stricte économie. Le Roi promit cependant d'approuver la construction d'une chapelle, de pièces sur pièces également, lorsque Beauharnois et Hocquart la jugeraient nécessaire. ${ }^{24}$

Or, au lieu de construire à la rivière Missisquoi une chapelle qui servirait en même temps aux colons français de la région, le gouverneur et l'intendant décidèrent d'établir une paroisse dans la seigneurie de Foucault, sur la rive est de la rivière Richelieu, établissement qui, selon eux, serait facilité par l'existence d'un moulin à blé dans cette seigneurie. Ils engagèrent le seigneur

21 Franquet, Voyages et mémoires sur le Canada, 96, 175.

22 Mémoire du Roi à Beauharnois et Hocquart, 24 mars 1744, AC, B 78.

23 Beauharnois et Hocquart au ministre, 12 octobre 1744 , AC, C11A, 81 .

24 Mémoire du Roi à Beauharnois et Hocquart, 28 avril 1745, AC, B 81 . 
du lieu à construire une chapelle en bois de 40 pieds de longueur sur 25 de largeur; et, comme il exigeait une contribution du roi, l'intendant lui promit l'aide de quelques-uns des ouvriers qu'il avait envoyés à Missisquoi et à la Rivière-du-Sud pour l'exploitation des bois de construction. ${ }^{25}$ Le 22 septembre 1744, par acte reçu par les notaires Boucault et Dulaurent, François Foucault fit don à Mgr de Pontbriand, évêque de Québec, d'une terre de deux arpents de front sur quarante de profondeur, pour y placer une église, un cimetière et, plus tard, un couvent des Sœurs de la Congrégation. ${ }^{26}$ L'église, ou plutôt la chapelle, fut seule construite, comme l'atteste Hocquart dans sa lettre au ministre, du 16 octobre 1745.27 Quant à la paroisse, le Roi en approuva la formation; ${ }^{28}$ et, pour l'entretien du missionnaire qui devait la desservir, le ministre autorisa Mgr de Pontbriand à prendre 400 livres sur les fonds que le Roi avait destinés aux curés usés. ${ }^{29}$ Mais la guerre de la Succession d'Autriche fit abandonner le projet de paroisse, ${ }^{30}$ et, après la paix d'Aix-la-Chapelle (1748), il n'en fut plus question. En 1751, la seigneurie de Foucault ne comptait pas plus de sept habitants. Lors de la guerre de la Conquête, à l'approche des Anglais, la chapelle construite par le seigneur fut jetée par terre, et ses matériaux servirent aux fortifications de l'Isle-aux-Noix. ${ }^{31}$ Elle était située à environ deux lieues du moulin de la seigneurie, ${ }^{32}$ dans la partie qui se trouve aujourd'hui en territoire américain, aux environs d'Alburg, Vermont.

La guerre terminée, on ne renonça pas au projet de fonder une mission pour les Abénakis de la rivière Missisquoi, et de grossir leur village pour le faire servir à barrer l'entrée du

25 Beauharnois et Hocquart au ministre, 12 octobre 1744, AC, C11A, 81. 26 BRH, 51 (1945) : 146-147.

27 \& Il y a une chapelle et un moulin sur la première» (la seigneurie de Foucault), AC, C11A, 83.

28 Mémoire du Roi à Beauharnois et Hocquart, 8 avril 1745, AC, B 81 .

${ }^{29}$ Le ministre à l'évêque de Québec, 12 mai 1745, AC, B 81.

$30 \mathrm{Mgr}$ de Pontbriand au ministre, 10 novembre 1746 , AC, C11A, 86. Auguste Gosselin, L'Eglise du Canada depuis Monseigneur de Laval jusqu'à la conquête, $3^{\mathrm{e}}$ partie (Québec, 1914), 227.

31 P.-G. Roy, Hommes et choses du Fort Saint-Frédéric, 324

32 《ournal militaire tenu par Nicolas Renaud d'Avène des Méloizes 》 (1759), RAPQ (1928-1929), 80. 
pays aux ennemis. Parlant des villages des Sauvages domiciliés, le Roi faisait écrire, le 30 avril 1749: "Il y en a encore un sixième dont l'établissement a été commencé à Missiskoui à l'entrée du lac Champlain, lequel doit être composé d'Abénakis et de Loups. ${ }^{33}$ De son côté, le gouverneur des Trois-Rivières, François Rigaud de Vaudreuil, apprenait au ministre, le 2 septembre de la même année: «Il y a encore une vingtaine de familles abénaquis dans une baie du lac Champlain, où on avait dessein d'établir un village à la tête du pays de ce côté-là, pour arrêter les courses de l'Anglais et leurs Sauvages d'Orange et les empêcher de pénétrer dans le pays. » ${ }^{34}$

Suivant les instructions qu'il avait reçues de Versailles, le gouverneur La Jonquière se fit rendre compte de l'état du village de Missisquoi, en 1749. On lui rapporta, et il écrit au ministre, que ce village était entièrement rétabli, ${ }^{35}$ et que les cabanes des Sauvages et la maison de leur missionnaire étaient en bon état. - S'il y avait eu une chapelle, il en aurait certainement fait mention. - Il ajoutait en post-scriptum: «J'oubliais de vous dire, Monseigneur, que le Révérend Père Lauverjat, Jésuite, qui était le missionnaire des dits Sauvages, est actuellement à la Pointe-à-la-Caille. » ${ }^{36}$ En effet, le Père Lauverjat avait dû quitter Missisquoi pour aller secourir spirituellement les 7 à 800 Sauvages d'Acadie, Abénakis, Micmacs et autres, qui, au commencement de la guerre, étaient venus se réfugier au Canada et s'étaient établis, moitié à la Pointe-à-la-Caille (Saint-Thomas de Montmagny) et moitié au saut de la Chaudière. ${ }^{37}$

Pour le peu de temps qu'il avait séjourné à Missisquoi, le Père Lauverjat n'en avait pas moins réussi à assurer la fidélité

${ }^{33}$ Mémoire du Roi pour servir d'instruction au $\mathrm{S}^{r}$ marquis de la Jonquière, 30 avril 1749 , AC, B 89 . - Les Loups des environs d'Orange vinrent s'établir avec leurs frères les Abénakis, en 1754, mais à SaintFrançois, non à Missisquoi. Cf. infra. 377.

34 Rigaud de Vaudreuil au ministre, 2 septembre 1749, BRH, 44 (1938) :

35 Il avait dû être évacué pendant la guerre, alors que les guerriers étaient presque continuellement en partis.

36 La Jonquière au ministre, 9 octobre $1749, \mathrm{AC}, \mathrm{C} 11 \mathrm{~A}, 93$.

37 L'intendant dut pourvoir à la subsistance de ces Sauvages, la chasse n'y suffisant pas. Il paraît qu'il en périt plus de la moitié. Lettre du Père Charlevoix, jésuite, à Monsieur Rouillé, 23 août 1749, Collection de manuscrits ... relatifs à la Nouvelle-France (4 vol., Québec, 1884), 3: 454. 
des Abénakis de l'endroit aux intérêts français. A l'été de 1744, Beauharnois envoya un officier leur chanter la guerre et leur offrir le collier et la hache. Ils le reçurent avec des démonstrations de zèle et d'attachement qui durèrent plusieurs jours.38 Et ils tinrent parole. Le village de Missisquoi, tout comme ceux de Saint-François et de Bécancour, mit ses guerriers au service des Français pour des expéditions de reconnaissance et pour les grandes campagnes militaires, pendant les deux guerres de la Succession d'Autriche et de la Conquête. Le dénombrement fait en 1736 par Maray de la Chauvinerie ne précise pas le nombre de ces guerriers: il donne le chiffre de 60 pour les Abénakis de Bécancour, et de 180 pour les Abénakis de Saint-François «y compris ceux de Michioukouy et les errants ${ }^{39}$ Mais l'officier que Beauharnois envoya leur chanter la guerre, en 1744, en compta 60 , «tous jeunes gens 》.

Hocquart écrivit au ministre, le 24 octobre 1746: « Les Abénaquis domiciliés ont été continuellement en petits partis sur les terres de la Nouvelle-Angleterre. Ils nous ont amené tout l'été des prisonniers et des chevelures. Vous verrez par l'Extrait de ce qui s'est passé tant à Québec qu'à Montréal le détail des incursions qu'ils ont faites sur les ennemis. " ${ }^{40}$ Un Journal de 1746 signale, à la date du 26 avril, qu'un parti de vingt guerriers abénakis de Missisquoi est allé du côté de Boston et a ramené des prisonniers et des chevelures; et, à la date du 24 mai, qu'un parti de huit Abénakis de Missisquoi a exécuté un coup du côté de Corlar (Schenectady) et est revenu avec des prisonniers et des chevelures. ${ }^{41}$ Le 7 mars 1747, les Abénakis de Missisquoi assistèrent, avec ceux de Saint-François et de Bécancour, à un conseil des nations sauvages tenu au Château de Montréal, où

38 Beauharnois et Hocquart au ministre, 12 octobre 1744, AC, C11A, 81.

39 \& Dénombrement des nations sauvages qui ont rapport au gouvernement de Canada; des guerriers de chaque nation avec les armoiries $1736 »$, BRH, 34 (1928): 541. Ce dénombrement a été fait par Maray de la Chauvinerie, interprète de la langue iroquoise. Henry Schoolcraft, Historical and statistical Information respecting the History, condition and prospects of the Indian Tribes of the United States (3 vol., Philadelphia, 1851-1857), 3: 553.

$40 \mathrm{AC}, \mathrm{C} 11 \mathrm{~A}, 85$.

41 Collection de manuscrits, 3 : 275, 278. 
on déclara la guerre aux Agniers. Ces derniers étaient venus faire des coups sur les côtes, au sud de Montréal. Comme on les croyait campés au-dessus de Châteauguay, le lieutenant SaintPierre partit, à la fin de mai 1747 , avec un détachement pour aller les surprendre. Huit Abénakis de Missisquoi l'accompagnaient. ${ }^{42}$

Au début du printemps de 1754, les Abénaquis de SaintFrançois et de Bécancour demandèrent au gouverneur Duquesne la permission de se venger sur les Anglais de deux chefs que ces derniers leur avaient tués du côté de Boston. Ils lui firent part en même temps d'un projet où il s'agissait de ramener dans leurs villages leurs frères les Loups établis dans le gouvernement d'Orange. Ces Loups leur avaient dit qu'ils en avaient assez de la domination anglaise et qu'ils seraient bien aises d'être de la prière. Duquesne leur répondit qu'il recevrait les Loups comme ses véritables enfants, qu'il ne pouvait s'opposer à leur juste vengeance, mais qu'il leur défendait de l'exercer contre les Anglais du gouvernement d'Orange. Les Abénakis de SaintFrançois exécutèrent leur dessein du côté de Boston. Mais ceux de Bécancour allèrent, malgré la défense du gouverneur, faire un coup du côté d'Orange. Les habitants, pris de terreur, se retirèrent dans la ville. Les Loups en profitèrent pour quitter leur village et se rendirent tous à Saint-François, à l'exception de cinq qui étaient à la chasse au moment du départ. ${ }^{43}$ L'année suivante, 100 Abénakis et Loups de Saint-François prenaient part à la campagne infructueuse de Dieskau au lac Saint-Sacrement. ${ }^{44}$

Tout comme ceux de Saint-François et de Bécancour, les Abénakis de Missisquoi étaient avec Montcalm au siège du fort de Chouaguen (ou Oswego), au sud du lac Ontario, en août $1756,{ }^{45}$ et à celui du fort George (ou William Henry), au lac Saint-

42 Ibid.: 327, 339.

43 Duquesne au ministre, 10 octobre 1754, Collection de manuscrits, $3: 515-516$.

44 Le Courtois des Bourbes à Le Courtois de Surlaville, 13 novembre 1755, BRH, 61 (1955) : 33.

45 Chaussegros de Léry, «Journal du siège du fort de Chouèguen 》, RAPQ (1926-1927), 403. 
Sacrement, en août 1757.46 Le 24 mars 1758, arriva un parti de 7 Abénakis de Missisquoi qui s'étaient rendus jusqu'à vingt lieues de Boston: ils avaient pillé et brûlé une maison de campagne, tué trois hommes et ramené une femme et ses deux enfants. ${ }^{47}$

Un mémoire anonyme, qui n'est pas daté mais qui est postérieur à 1758, dit que «d'autres Abénaquis établis au fond de la baie de Missisquoi, dans le lac Champlain, se sont depuis le commencement de cette guerre retirés à Saint-François et à Bécancour ».48 Il s'agit sans doute de la campagne de 1759. On sait qu'au lieu d'affronter l'armée anglaise du lac Champlain, par trop supérieure à la sienne, Bourlamaque fit sauter les forts Carillon et Saint-Frédéric, et vint se retrancher dans l'Isle-auxNoix, à l'entrée de la rivière Richelieu. Le village abénakis de Missisquoi s'en trouva menacé. Le moulin à scie bâti par Levasseur de Néré fut abandonné, et sa ferrure enterrée. ${ }^{49}$ Les guerriers toutefois, et même leurs femmes, demeurèrent dans les environs de l'Isle-aux-Noix, et participèrent aux opérations de reconnaissance de l'armée de Bourlamaque. Ils s'adressèrent, pour fins religieuses, à l'aumônier du fort Saint-Jean. Le 9 août 1759 , le Père Elzéar Maugé, récollet, baptisa « Athanase, né hier au soir, fils de Jean-Baptiste Onision sauvage abénakis du village de Missisquois $\gg .{ }^{50}$

Thomas ChaRland, O.P. vice-président de l'Institut d'histoire de l'Amérique française

46 Journal du marquis de Montcalm (Québec, 1895), 242; « Journal de M. de Bougainville », RAPQ (1923-1924), 278, 287.

47 Journal de Montcalm, 341; Journal de Bougainville, 317; Montcalm à Bougainville, 28 mars 1758, Collection des manuscrits du maréchal de Lévis (12 vol., Montréal et Québec, 1889-1895), $5: 217$. 412.

48 «émoire sur les forts de la Nouvelle-France», BRH, 37 (1931):

49 Speech of the Misiskouy Indians ....

50 Copie du registre du fort Saint-Jean, 1757-1760, Archives canadiennes, M, 200. - Les Abénakis de Missisquoi s'étaient déjà adressés à l'aumônier de ce fort. J'ai relevé dans le registre 4 baptêmes et 2 sépultures d'Abénakis pour les années 1757 et 1758 . Un de ces baptêmes, celui du 17 juillet 1758, fut fait par le Père Audran, s.j., \& du consentement du Père Denis Baron, récollet $\gg$. 